\title{
Dynamical analysis of an SIRS network model with direct immunization and infective vector
}

\author{
Rongzhong $\mathrm{Yu}^{1 *}{ }^{*}$, Kezan $\mathrm{Li}^{2}$, Baidi Chen ${ }^{1}$ and Dingqin Shi ${ }^{1}$
}

${ }^{\text {*Correspondence: }}$ yurongzhong@163.com

${ }^{1}$ College of Sciences, Jiujiang University, Qianjin East Road, Jiujiang, 332005, China Full list of author information is available at the end of the article

\begin{abstract}
With the awareness of risk in infective disease spreading, healthy individuals (the susceptible ones) will take some measures to acquire temporary immunity. This paper addresses an SIRS model with direct immunization and an infective vector in complex networks and performs the dynamical analysis for this model. By theoretical analysis, we obtain the epidemic threshold $\lambda_{c}$ and prove that if infection rate $\lambda<\lambda_{c}$, the disease-free equilibrium is globally asymptotically stable; if $\lambda>\lambda_{c}$, there exists a unique endemic equilibrium, and it is globally attractive. These theoretical results are confirmed by numerical simulations.
\end{abstract}

Keywords: epidemic threshold; dynamical analysis; epidemic equilibrium; SIRS model; global attraction

\section{Introduction}

In recent years, many epidemic models on complex networks, such as SIS (susceptibleinfected-susceptible) [1-8] and SIR (susceptible-infected-removed) [9-16] and so on, have been widely studied by researchers from different subjects. Classical studies have revealed that there is an epidemic threshold $\lambda_{c}$ for an epidemic model on homogeneous networks, below which the disease will die out; otherwise there will exist a persistence. However, Pastor-Satorras and Vespignani further showed a striking result that the epidemic threshold $\lambda_{c}$ will vanish for a heterogenous network with sufficiently large sizes [1, 2, 4].

In fact, apart from the human behavior [17, 18] and the external environment [19], the infection vector (e.g., mosquitoes) may also play an important role in epidemic transmission $[3,20,21]$. The infective vector generally acts as a carrier of an infective disease and can transmit it to a human. By considering the disease spreading on a human network caused by an infection vector, Cooke and Busenberg [22, 23] have addressed some epidemic compartment models. As we known, some diseases spread not only by contacts between people and infected vectors but also by blood contacts within human. By noting this fact, Shi et al. [21] proposed a new SIS model with an infective medium on complex networks, which models the spread of a class of infectious diseases. Then a modified SIS model is proposed in [3] by assuming that the human contacts can be considered as a scale-free network, but the infective media may contact a person without any selectivity. The epidemic threshold and the stability of endemic equilibrium are investigated theoretically. A more general modified SIS model with an infective medium on complex networks was introduced in [20], and the authors investigated the global attraction of endemic equilibrium by the basic reproduction number $R_{0}$. However, the direct relation between the 
epidemic threshold $\lambda_{c}$ in [3] and the basic reproduction number $R_{0}$ in [20] has not been revealed.

In the real world, with the awareness of an infectious disease spreading, some healthy individuals will usually take some protective measures (e.g., vaccine inoculation) to acquire temporary immunity. By investigating an SIRS epidemic model [24] with direct immunization on complex networks, the result shows that the direct immunization can increase the epidemic threshold and reduce the prevalence of infectious disease.

In this paper, we propose a new SIRS model with direct immunization and an infective vector on complex networks. We get the epidemic threshold $\lambda_{c}$, below which the diseasefree equilibrium is globally stable; otherwise, the disease-free equilibrium is unstable and a unique endemic equilibrium exists, and it is also globally attractive. More importantly, according to our method, one can directly determine the relation between the epidemic threshold and the basic reproduction number.

The rest of the paper is organized as follows. In Section 2, we propose a new SIRS model with direct immunization and an infective vector on complex networks. Section 3 analyzes the dynamics of the model and shows some theoretical results. Some numerical simulations are performed to confirm our theoretical predictions in Section 4.

\section{The model}

Based on the real mechanism of some relevant epidemic networks, our model will be constructed with the following context:

- Two infection mechanisms: the disease spreads not only by contacts between individuals, but also by contacts between individuals and infective vectors.

- Two removed ways: (i) with awareness of risk infective disease spreading, some health individuals may make some protective measures (vaccination) to acquire temporary immunity; (ii) an infected individual becoming a removed individual after cure may acquire temporary immunity.

In addition, we further suppose that the individuals' contacts can be treated as heterogeneous, but the contacts between individuals and vectors can be considered as homogeneous. This assumption results from the selective contacts in a human network and non-selective contacts between people and vectors [3, 20].

Let $S_{k}(t), I_{k}(t)$ and $R_{k}(t)$ be the densities of susceptible, infected and removed nodes with degree $k$ at time $t$ respectively, and let $V(t)$ be the density of the infective medium at time $t$. Let $\rho(t)=\sum p(k) I_{k}(t)$ denote the density of infected individuals on the network, and $\Theta$ represents the probability that a randomly chosen link emanating from a node of degree $k$ leads to infected nodes. In this paper, we consider the situation of uncorrelated networks, then $\Theta$ can be written as $\Theta=\frac{1}{\langle k\rangle} \sum_{k^{\prime}} k^{\prime} p\left(k^{\prime}\right) I_{k^{\prime}}(t)[1,2]$, where $p(k)$ denotes the degree distribution of the network, $\langle k\rangle=\sum k p(k)$ is its average degree.

We assume that the susceptible nodes become the removed nodes with rate $\alpha$ for acquiring temporary immunity. At the same time, each susceptible (health) node is infected with rates $\lambda$ and $\gamma_{1}$ if it is contacted to infected nodes and infective vectors, respectively. Infected nodes are cured with rate $\beta$ and removed nodes again become susceptible with rate $\delta$ for immunization-lost. Health vectors are infected with rate $\gamma_{2}$ if they are contacted to infected individuals and infected vectors recover with rate $\xi$.

In the real world, an epidemic always occurs on a finite network [4] even though the size of the network is very large. Hence, we consider disease transmission in a finite population in this paper, and let $n$ be the maximum degree. Then, neglecting of contact duration, 
the proposed SIRS propagation model can be described by the following differential equations:

$$
\left\{\begin{array}{l}
\frac{d S_{k}(t)}{d t}=-\lambda k S_{k}(t) \Theta+\delta R_{k}(t)-\gamma_{1} V(t) S_{k}(t)-\alpha S_{k}(t) \\
\frac{d I_{k}(t)}{d t}=\lambda k S_{k}(t) \Theta-\beta I_{k}(t)+\gamma_{1} V(t) S_{k}(t) \\
\frac{d R_{k}(t)}{d t}=\beta I_{k}(t)-\delta R_{k}(t)+\alpha S_{k}(t) \\
\frac{d V(t)}{d t}=-\xi V(t)+\gamma_{2}(1-V(t)) \rho(t)
\end{array}\right.
$$

Without loss of generality, assume that the infective vector has unit recovery, i.e., $\xi=1$. In addition, the variables $S_{k}(t), I_{k}(t), R_{k}(t)$ satisfy the normalization condition $S_{k}(t)+I_{k}(t)+$ $R_{k}(t)=1$. Then Eq. (1) can be rewritten as

$$
\left\{\begin{array}{l}
\frac{d I_{k}(t)}{d t}=\left[\lambda k \Theta+\gamma_{1} V(t)\right]\left[1-I_{k}(t)-R_{k}(t)\right]-\beta I_{k}(t) \\
\frac{d R_{k}(t)}{d t}=(\beta-\alpha) I_{k}(t)-(\alpha+\delta) R_{k}(t)+\alpha \\
\frac{d V(t)}{d t}=-V(t)+\gamma_{2}(1-V(t)) \rho(t)
\end{array}\right.
$$

\section{Epidemic threshold and global analysis}

\subsection{Epidemic threshold}

Theorem 1 Let $\lambda_{c}=\frac{\left[\beta(\alpha+\delta)-\delta \gamma_{1} \gamma_{2}\right] \beta(\alpha+\delta)\langle k\rangle}{\delta^{2} \gamma_{1} \gamma_{2}\left(\langle k\rangle^{2}-\left\langle k^{2}\right\rangle\right)+\delta \beta(\delta+\alpha)\left\langle k^{2}\right\rangle}$ and $\beta-\frac{\delta}{\alpha+\delta} \gamma_{1} \gamma_{2}>0$, if $\lambda>\lambda_{c}$, then one and only one endemic equilibrium solution of system (2) exists, i.e., the epidemic propagation may outbreak on complex networks.

Proof By letting the right-hand side of system (2) be zero, we have

$$
\begin{aligned}
& \beta I_{k}(t)=\left[\lambda k \Theta+\gamma_{1} V(t)\right]\left[1-R_{k}(t)-I_{k}(t)\right], \\
& (\beta-\alpha) I_{k}(t)-(\alpha+\delta) R_{k}(t)+\alpha=0
\end{aligned}
$$

and

$$
V(t)=\frac{\gamma_{2} \rho(t)}{1+\gamma_{2} \rho(t)}
$$

Substituting (4) into (3), one obtains

$$
\beta I_{k}(t)=\left[\lambda k \Theta+\gamma_{1} V(t)\right]\left[\frac{\delta}{\delta+\alpha}-\frac{\beta+\delta}{\delta+\alpha} I_{k}(t)\right] .
$$

Then substituting (5) into (6), we have

$$
I_{k}(t)=\frac{\delta\left[\lambda k \Theta+\lambda k \gamma_{2} \rho(t) \Theta+\gamma_{1} \gamma_{2} \rho(t)\right]}{\beta(\delta+\alpha)\left[1+\gamma_{2} \rho(t)\right]+(\beta+\delta)\left[\lambda k \Theta+\lambda k \gamma_{2} \rho(t) \Theta+\gamma_{1} \gamma_{2} \rho(t)\right]} .
$$

Let

$$
\mathcal{F}_{1}=\delta\left[\lambda k \Theta+\lambda k \gamma_{2} \rho(t) \Theta+\gamma_{1} \gamma_{2} \rho(t)\right]
$$

and

$$
\mathcal{F}_{2}=\beta(\delta+\alpha)\left[1+\gamma_{2} \rho(t)\right]+(\beta+\delta)\left[\lambda k \Theta+\lambda k \gamma_{2} \rho(t) \Theta+\gamma_{1} \gamma_{2} \rho(t)\right] .
$$


Then one has a self-consistency equation as follows:

$$
\Theta(t)=\frac{1}{\langle k\rangle} \sum_{k} k p(k) I_{k}(t)=\frac{1}{\langle k\rangle}\left\langle k \frac{\mathcal{F}_{1}}{\mathcal{F}_{2}}\right\rangle \equiv \mathcal{F}(\Theta) .
$$

It is obvious that $\Theta(t)=0$ is a trivial solution to Eq. (8). What we are interested in is the condition under which the epidemic propagation outbreaks. Since $\Theta \in[0,1], \mathcal{F}(0)=0$ and $\mathcal{F}(\Theta) \in[0,1)$, then Eq. (8) must have a non-trivial solution if $\left.\frac{d \mathcal{F}}{d \Theta}\right|_{\Theta=0}>1$.

By computing the following expression

$$
\left.\frac{d \mathcal{F}}{d \Theta}\right|_{\Theta=0}=\frac{1}{\langle k\rangle}\left\langle\left. k \frac{\left(\frac{\partial \mathcal{F}_{1}}{\partial \rho} \cdot \frac{d \rho}{d \Theta}+\frac{\partial \mathcal{F}_{1}}{\partial \Theta}\right) \mathcal{F}_{2}-\left(\frac{\partial \mathcal{F}_{2}}{\partial \rho} \cdot \frac{d \rho}{d \Theta}+\frac{\partial \mathcal{F}_{2}}{\partial \Theta}\right) \mathcal{F}_{1}}{\mathcal{F}_{2}^{2}}\right|_{\Theta=0}>1,\right.
$$

we have the epidemic threshold $\lambda_{c}$ taking the following expression in the case where $\beta-$ $\frac{\delta}{\alpha+\delta} \gamma_{1} \gamma_{2}>0$,

$$
\lambda_{c}=\frac{\left[\beta(\alpha+\delta)-\delta \gamma_{1} \gamma_{2}\right] \beta(\alpha+\delta)\langle k\rangle}{\delta^{2} \gamma_{1} \gamma_{2}\left(\langle k\rangle^{2}-\left\langle k^{2}\right\rangle\right)+\delta \beta(\delta+\alpha)\left\langle k^{2}\right\rangle} .
$$

Inequality (9) holds if and only if $\lambda>\lambda_{c}$. Furthermore, we will ascertain the uniqueness of endemic equilibrium in a similar way as paper [20]. Assume that $I=\left(I_{1}, I_{2}, \ldots, I_{n}, V\right)$ and $I^{*}=\left(I_{1}^{*}, I_{2}^{*}, \ldots, I_{n}^{*}, V^{*}\right)$ are two different roots to Eqs. (5) and (6). Let

$$
\eta=\max \left\{\max _{k=1, \ldots, n}\left\{\frac{I_{k}}{I_{k}^{*}}\right\}, \frac{V}{V^{*}}\right\} .
$$

Moreover, assume that $\eta>1$ without loss of generality. We will complete the proof in two cases as follows.

Case 1: If there exists a natural number $k_{0} \in\{1,2, \ldots, n\}$ such that $\eta=\frac{I_{k_{0}}}{I_{k_{0}}^{*}}$, then we, by (6), have

$$
\begin{aligned}
& \beta I_{k_{0}}=\left[\lambda k_{0} \Theta(I)+\gamma_{1} V\right]\left[\frac{\delta}{\delta+\alpha}-\frac{\beta+\delta}{\delta+\alpha} I_{k_{0}}\right], \\
& \beta I_{k_{0}}^{*}=\left[\lambda k_{0} \Theta\left(I^{*}\right)+\gamma_{1} V^{*}\right]\left[\frac{\delta}{\delta+\alpha}-\frac{\beta+\delta}{\delta+\alpha} I_{k_{0}}^{*}\right],
\end{aligned}
$$

where $\Theta(I)=\frac{1}{\langle k\rangle} \sum k p(k) I_{k}$ and $\Theta\left(I^{*}\right)=\frac{1}{\langle k\rangle} \sum k p(k) I_{k}^{*}$. From (10) and (11), we can obtain

$$
\left[\lambda k_{0} \Theta(I)+\gamma_{1} V\right]\left[\frac{\delta}{\delta+\alpha}-\frac{\beta+\delta}{\delta+\alpha} I_{k_{0}}\right]=\eta \cdot\left[\lambda k_{0} \Theta\left(I^{*}\right)+\gamma_{1} V^{*}\right]\left[\frac{\delta}{\delta+\alpha}-\frac{\beta+\delta}{\delta+\alpha} I_{k_{0}}^{*}\right]
$$

while we have the following inequalities according to the definition of $\eta$ :

$$
\begin{aligned}
& \eta \cdot\left[\lambda k_{0} \Theta\left(I^{*}\right)+\gamma_{1} V^{*}\right]=\lambda k_{0} \Theta\left(I^{*}\right) \cdot \eta+\gamma_{1} V^{*} \cdot \eta>\lambda k_{0} \Theta(I)+\gamma_{1} V, \\
& \frac{\delta}{\delta+\alpha}-\frac{\beta+\delta}{\delta+\alpha} I_{k_{0}}^{*}>\frac{\delta}{\delta+\alpha}-\frac{\beta+\delta}{\delta+\alpha} I_{k_{0}} .
\end{aligned}
$$

It is obvious that (13) and (14) contradict (12). 
Case 2: If $\eta=\frac{V}{V^{*}}$, according to (5), then we have

$$
\begin{aligned}
& V=(1-V) \gamma_{2} \rho(I), \\
& V^{*}=\left(1-V^{*}\right) \gamma_{2} \rho\left(I^{*}\right),
\end{aligned}
$$

where $\rho(I)=\sum p(k) I_{k}$ and $\rho\left(I^{*}\right)=\sum p(k) I_{k}^{*}$. From (15), (16) and the definition of $\eta$, one obtains

$$
(1-V) \gamma_{2} \rho(I)=\left(1-V^{*}\right) \gamma_{2} \rho\left(I^{*}\right) \cdot \eta>(1-V) \gamma_{2} \sum p(k)\left(I_{k}^{*} \cdot \eta\right)>(1-V) \gamma_{2} \rho(I) .
$$

A contradiction appears. In conclusion, when $\lambda>\lambda_{c}$, one and only one endemic equilibrium solution exists for system (1).

Letting $\tau=\frac{\delta}{\delta+\alpha}$, the epidemic threshold $\lambda_{c}$ can be rewritten as

$$
\lambda_{c}=\frac{\left[\beta-\tau \gamma_{1} \gamma_{2}\right] \beta\langle k\rangle}{\tau^{2} \gamma_{1} \gamma_{2}\left(\langle k\rangle^{2}-\left\langle k^{2}\right\rangle\right)+\tau \beta\left\langle k^{2}\right\rangle} .
$$

Note that the assumption that $\beta-\tau \gamma_{1} \gamma_{2}>0$, which is a default condition below, ensures that the threshold $\lambda_{c}$ is larger than zero for a finite size network. One can see that the critical threshold vanishes for a scale-free network with sufficiently large sizes, which agrees with the previous papers $[1,2,4]$.

The reader should find that the model parameters are general in this model. If $\gamma_{1}$ or $\gamma_{2}$ vanishes, the proposed model may become an SIRS model with direct immunization via one infection mechanism (contacts between individuals), and the epidemic $\lambda_{c}=\frac{\beta\langle k\rangle}{\tau\left\langle k^{2}\right\rangle}$ which agrees with the one of paper [24]. In addition, the proposed model may become one SIRS model with an infection vector via two infection mechanisms when $\alpha=0$. And the epidemic threshold $\lambda_{c}=\frac{\left[\beta-\gamma_{1} \gamma_{2}\right] \beta\langle k\rangle}{\gamma_{1} \gamma_{2}\left(\langle k\rangle^{2}-\left\langle k^{2}\right\rangle\right)+\beta\left\langle k^{2}\right\rangle}$, especially, $\lambda_{c}=\frac{\left[1-\gamma_{1} \gamma_{2}\right]\langle k\rangle}{\gamma_{1} \gamma_{2}\left(\langle k\rangle^{2}-\left\langle k^{2}\right\rangle\right)+\left\langle k^{2}\right\rangle}$ if $\beta=1$, which is in accordance with the one of paper [3].

\subsection{Global stability of disease-free equilibrium}

Theorem 2 For system (1), let $\lambda_{c}$ be the epidemic threshold defined as (18). If $\lambda<\lambda_{c}$, then the disease-free equilibrium is globally asymptotically stable. Otherwise, there exists a unique endemic equilibrium.

Proof For convenience, system (1) can be rewritten as follows:

$$
\left\{\begin{array}{l}
\frac{d S_{k}(t)}{d t}=-\left[\lambda k \Theta+\gamma_{1} V(t)+\delta+\alpha\right] S_{k}(t)-\delta I_{k}(t)+\delta, \\
\frac{d I_{k}(t)}{d t}=\left[\lambda k \Theta+\gamma_{1} V(t)\right] S_{k}(t)-\beta I_{k}(t), \\
\frac{d V(t)}{d t}=-V(t)+\gamma_{2}(1-V(t)) \rho(t) .
\end{array}\right.
$$

The disease-free equilibrium of Eq. (19) is $S_{k}=\frac{\delta}{\delta+\alpha}=\tau, I_{k}=0, V=0, k=1,2, \ldots, n$. Moreover, the Jacobian matrix at disease-free equilibrium can be represented as

$$
J=\left[\begin{array}{ll}
D & F \\
0 & M
\end{array}\right],
$$


where $D=-(\delta+\alpha) E_{n}$, and $E_{n}$ is an $n$th identity matrix, $F$ and $M$ are $n \times(n+1)$ and $(n+$ 1) $\times(n+1)$ matrices taking the following forms, respectively:

$$
\begin{aligned}
& F=\left[\begin{array}{ccccc}
-\frac{\tau \lambda}{\langle k\rangle} p(1)-\delta & -\frac{\tau \lambda}{\langle k\rangle} 2 \cdot p(2) & \ldots & -\frac{\tau \lambda}{\langle k\rangle} n \cdot p(n) & -\gamma_{1} \tau \\
-\frac{\tau \lambda}{\langle k\rangle} 2 \cdot p(1) & -\frac{\tau \lambda}{\langle k\rangle} 2^{2} \cdot p(2)-\delta & \ldots & -\frac{\tau \lambda}{\langle k\rangle} 2 n \cdot p(n) & -\gamma_{1} \tau \\
\vdots & \vdots & \ddots & \vdots & \vdots \\
-\frac{\tau \lambda}{\langle k\rangle} n \cdot p(1) & -\frac{\tau \lambda}{\langle k\rangle} n \cdot 2 \cdot p(2) & \cdots & -\frac{\tau \lambda}{\langle k\rangle} n^{2} \cdot p(n)-\delta & -\gamma_{1} \tau
\end{array}\right], \\
& M=\left[\begin{array}{ccccc}
\frac{\tau \lambda}{\langle k} p(1)-\beta & \frac{\tau \lambda}{\langle k\rangle} 2 \cdot p(2) & \ldots & \frac{\tau \lambda}{\langle k\rangle} n \cdot p(n) & \gamma_{1} \tau \\
\frac{\tau \lambda}{\langle k\rangle} 2 \cdot p(1) & \frac{\tau \lambda}{\langle k\rangle} 2^{2} \cdot p(2)-\beta & \ldots & \frac{\tau \lambda}{\langle k\rangle} 2 n \cdot p(n) & \gamma_{1} \tau \\
\vdots & \vdots & \ddots & \vdots & \vdots \\
\frac{\tau \lambda}{\langle k\rangle} n \cdot p(1) & \frac{\tau \lambda}{\langle k\rangle} n \cdot 2 \cdot p(2) & \cdots & \frac{\tau \lambda}{\langle k\rangle} n^{2} \cdot p(n)-\beta & \gamma_{1} \tau \\
\gamma_{2} p(1) & \gamma_{2} p(2) & \cdots & \gamma_{2} p(n) & -1
\end{array}\right] .
\end{aligned}
$$

It is obvious that all of eigenvalues of $J$ have negative part if and only if all of eigenvalues of $M$ have negative part. Denote $A=M-\mu \cdot E$ and $v=\mu+\beta$, where $E$ is an $(n+1)$-order unit matrix, and $\mu$ is eigenvalue, then

$$
\operatorname{det} A=\left|\begin{array}{ccccc}
\frac{\tau \lambda}{\langle k} p(1)-v & \frac{\tau \lambda}{\langle k\rangle} 2 \cdot p(2) & \cdots & \frac{\tau \lambda}{\langle k\rangle} n \cdot p(n) & \gamma_{1} \tau \\
\frac{\tau \lambda}{\langle k\rangle} 2 \cdot p(1) & \frac{\tau \lambda}{\langle k\rangle} 2^{2} \cdot p(2)-v & \cdots & \frac{\tau \lambda}{\langle k\rangle} 2 n \cdot p(n) & \gamma_{1} \tau \\
\vdots & \vdots & \ddots & \vdots & \vdots \\
\frac{\tau \lambda}{\langle k\rangle} n \cdot p(1) & \frac{\tau \lambda}{\langle k\rangle} n \cdot 2 \cdot p(2) & \cdots & \frac{\tau \lambda}{\langle k\rangle} n^{2} \cdot p(n)-v & \gamma_{1} \tau \\
\gamma_{2} p(1) & \gamma_{2} p(2) & \cdots & \gamma_{2} p(n) & -v-(1-\beta)
\end{array}\right| .
$$

It follows that all of eigenvalues of $M$ have negative real part if and only if all of roots of the characteristic polynomial $\operatorname{det} A=0$ have real part of at most $\beta$. By some elementary transformations, one obtains that the issue above holds if and only all of roots of the following cubic polynomial have real part of at most $\beta$ :

$$
g(v)=v^{3}-a_{2} v^{2}-a_{1} v+a_{0}=0,
$$

where

$$
\begin{aligned}
& a_{2}=\frac{\tau \lambda\left\langle k^{2}\right\rangle-(1-\beta)\langle k\rangle}{\langle k\rangle}, \\
& a_{1}=\frac{(1-\beta) \tau \lambda\left\langle k^{2}\right\rangle+\gamma_{1} \gamma_{2} \tau\langle k\rangle}{\langle k\rangle}, \\
& a_{0}=\frac{\gamma_{1} \gamma_{2} \tau^{2} \lambda\left[\left\langle h k^{2}\right\rangle-\langle k\rangle^{2}\right]}{\langle k\rangle} .
\end{aligned}
$$

It is easy to obtain that

$$
\begin{aligned}
& \lim _{\nu \rightarrow-\infty} g(v)=-\infty, \quad \lim _{\nu \rightarrow+\infty} g(v)=+\infty, \\
& g(0)=\frac{\gamma_{1} \gamma_{2} \tau^{2} \lambda\left[\left\langle k^{2}\right\rangle-\langle k\rangle^{2}\right]}{\langle k\rangle}>0, \quad g\left(\lambda \tau \frac{\left\langle k^{2}\right\rangle}{\langle k\rangle}\right)=-\gamma_{1} \gamma_{2} \tau^{2} \lambda\langle k\rangle<0,
\end{aligned}
$$


since $\left\langle k^{2}\right\rangle \gg\langle k\rangle^{2}$ for a sufficiently large network. We will investigate the roots to the cubic equation (21) below:

$$
g(\beta)=\beta^{2}-\beta \tau \lambda \frac{\left\langle k^{2}\right\rangle}{\langle k\rangle}-\gamma_{1} \gamma_{2} \tau \beta+\gamma_{1} \gamma_{2} \tau^{2} \lambda \frac{\left\langle k^{2}\right\rangle-\langle k\rangle^{2}}{\langle k\rangle} \begin{cases}>0, & \text { if } \lambda<\lambda_{c} \\ <0, & \text { if } \lambda>\lambda_{c} .\end{cases}
$$

If $\lambda<\lambda_{c}$, then $\lambda \tau \frac{\left\langle k^{2}\right\rangle}{\langle k\rangle}<\beta$ since $\lambda_{c}<\frac{\beta\langle k\rangle}{\tau\left\langle k^{2}\right\rangle}$. It implies that the cubic equation (21) has three roots $v_{1}, v_{2}$ and $v_{3}$ satisfying

$$
-\infty<v_{1}<0<v_{2}<\lambda \tau \frac{\left\langle k^{2}\right\rangle}{\langle k\rangle}<v_{3}<\beta
$$

If $\lambda>\lambda_{c}$, then the cubic equation (21) has three roots satisfying

$$
-\infty<\nu_{1}<0<\nu_{2}<\beta<\nu_{3}<+\infty
$$

In a word, there exists a unique positive eigenvalue of $J$ if and only if $\lambda>\lambda_{c}$, below which the unique epidemic equilibrium exists. Otherwise all real-valued eigenvalues of $J$ are negative, this implies that the disease-free equilibrium is globally stable according to Lemma 1 in paper [14].

In paper [20], the authors follow the concepts of next-generation matrix (NGM) to give a threshold - the basic reproduction number $R_{0}$, by which the global stability of a modified SIS model is studied. The NGM is a matrix that relates the numbers of newly infected individuals in various categories in consecutive generation, and the basic reproduction number $R_{0}$ is the spectral radius of the NGM (refer to the papers [25, 26] for details). However, the direct relationship between the epidemic threshold and the basic reproduction number is not clearly revealed. In fact, we can reveal that $\lambda=\lambda_{c}$ if and only if $R_{0}=1$ by the same way as above.

\subsection{Global attraction of endemic equilibrium}

In this part, we show a proposition and prove the global attraction of the endemic equilibrium by the same way as the one in [3, 8]. Inequalities (27) and (28) in Proposition 2 are helpful to prove the main result (Theorem 3 ).

Proposition 1 Suppose that $\alpha \geq \beta$ and the solution $I_{k}(t)$ of system (2) satisfies $\lim \sup _{t \rightarrow \infty} I_{k}(t) \leq U_{k}, \liminf _{t \rightarrow \infty} I_{k}(t) \geq L_{k}$, then

$$
\limsup _{t \rightarrow \infty} V(t) \leq \frac{\gamma_{2}\left\langle U_{k}\right\rangle}{1+\gamma_{2}\left\langle U_{k}\right\rangle}, \quad \liminf _{t \rightarrow \infty} V(t) \geq \frac{\gamma_{2}\left\langle L_{k}\right\rangle}{1+\gamma_{2}\left\langle L_{k}\right\rangle}
$$

and

$$
\limsup _{t \rightarrow \infty}\left(1-R_{k}(t)\right) \leq \frac{(\alpha-\beta) U_{k}+\delta}{\alpha+\delta}, \quad \liminf _{t \rightarrow \infty}\left(1-R_{k}(t)\right) \geq \frac{(\alpha-\beta) L_{k}+\delta}{\alpha+\delta} .
$$

Proof Without loss of generality, we only verify the upper limit inequalities. From $\lim \sup _{t \rightarrow \infty} I_{k}(t) \leq U_{k}$, we obtain that for any $\epsilon>0$, there exists $\tau_{0}>0$ such that $I_{k}(t) \leq$ 
$U_{k}+\epsilon$ for $t>\tau_{0}$. It follows that

$$
\begin{aligned}
\frac{d V(t)}{d t} & =-V(t)+\gamma_{2}(1-V(t)) \rho(t) \leq-V(t)+\gamma_{2}(1-V(t))\left\langle U_{k}+\epsilon\right\rangle \\
& =-\left(1+\gamma_{2}\left\langle U_{k}+\epsilon\right\rangle\right) V(t)+\gamma_{2}\left\langle U_{k}+\epsilon\right\rangle
\end{aligned}
$$

for $t>\tau_{0}$. Therefore, the following inequality holds since $\epsilon>0$ is arbitrarily small:

$$
\limsup _{t \rightarrow \infty} V(t) \leq \frac{\gamma_{2}\left\langle U_{k}\right\rangle}{1+\gamma_{2}\left\langle U_{k}\right\rangle}
$$

At the same time, it follows from the second equation of system (2) and $\alpha \geq \beta$ that

$$
\begin{aligned}
\frac{d\left(1-R_{k}(t)\right)}{d t} & =-(\alpha+\delta)\left(1-R_{k}(t)\right)+(\alpha-\beta) I_{k}(t)+\delta \\
& \leq-(\alpha+\delta)\left(1-R_{k}(t)\right)+(\alpha-\beta)\left(U_{k}+\epsilon\right)+\delta
\end{aligned}
$$

Consequently,

$$
\limsup _{t \rightarrow \infty}\left(1-R_{k}(t)\right) \leq \frac{(\alpha-\beta) U_{k}+\delta}{\alpha+\delta} .
$$

Proposition 2 Suppose that $\alpha \geq \beta$ and the solution $I_{k}(t)$ of system (2) satisfies $\limsup \operatorname{su}_{t \rightarrow \infty} I_{k}(t) \leq U_{k}, \liminf _{t \rightarrow \infty} I_{k}(t) \geq L_{k}$, then

$$
\begin{aligned}
& \limsup _{t \rightarrow \infty} I_{k}(t) \leq \frac{\left[\lambda \frac{k}{\langle k\rangle}\left\langle k U_{k}\right\rangle+\frac{\gamma_{1} \gamma_{2}\left\langle U_{k}\right\rangle}{1+\gamma_{2}\left\langle U_{k}\right\rangle}\right]\left[\frac{\delta+(\alpha-\beta) U_{k}}{\alpha+\delta}\right]}{\beta+\lambda \frac{k}{\langle k\rangle}\left\langle k U_{k}\right\rangle+\frac{\gamma_{1} \gamma_{2}\left\langle U_{k}\right\rangle}{1+\gamma_{2}\left\langle U_{k}\right\rangle}}, \\
& \liminf _{t \rightarrow \infty} I_{k}(t) \geq \frac{\left[\lambda \frac{k}{\langle k\rangle}\left\langle k L_{k}\right\rangle+\frac{\gamma_{1} \gamma_{2}\left\langle L_{k}\right\rangle}{1+\gamma_{2}\left\langle L_{k}\right\rangle}\right]\left[\frac{\delta+(\alpha-\beta) L_{k}}{\alpha+\delta}\right]}{\beta+\lambda \frac{k}{\langle k\rangle}\left\langle k L_{k}\right\rangle+\frac{\gamma_{1} \gamma_{2}\left\langle L_{k}\right\rangle}{1+\gamma_{2}\left\langle L_{k}\right\rangle}} .
\end{aligned}
$$

Proof From $\limsup \sup _{t \rightarrow \infty} I_{k}(t) \leq U_{k}$ and Proposition 1, one obtains that for any $\epsilon>0$, there exists large enough $\tau$ such that the following inequalities hold for $t>\tau$ :

$$
\begin{aligned}
& \Theta(t, k) \leq \frac{\left\langle k\left(U_{k}+\epsilon\right)\right\rangle}{\langle k\rangle}, \\
& V(t) \leq \frac{\gamma_{2} U_{k}}{1+\gamma_{2} U_{k}}+\epsilon, \\
& 1-R_{k}(t) \leq \frac{(\alpha-\beta) U_{k}+\delta}{\alpha+\delta}+\epsilon .
\end{aligned}
$$

Considering the first equation of system (2), for $t>\tau$, it follows from (29), (30) and (31) that

$$
\begin{aligned}
\frac{d I_{k}(t)}{d t}= & {\left[\lambda k \Theta(t, k)+\gamma_{1} V(t)\right]\left[1-I_{k}(t)-R_{k}(t)\right]-\beta I_{k}(t) } \\
\leq & {\left[\lambda \frac{k}{\langle k\rangle}\left\langle k\left(U_{k}+\epsilon\right)\right\rangle+\gamma_{1}\left(\frac{\gamma_{2} U_{k}}{1+\gamma_{2} U_{k}}+\epsilon\right)\right] } \\
& \times\left[\frac{(\alpha-\beta) U_{k}+\delta}{\alpha+\delta}+\epsilon-I_{k}(t)\right]-\beta I_{k}(t)
\end{aligned}
$$




$$
\begin{aligned}
= & -\left[\beta+\lambda \frac{k}{\langle k\rangle}\left\langle k\left(U_{k}+\epsilon\right)\right\rangle+\gamma_{1}\left(\frac{\gamma_{2} U_{k}}{1+\gamma_{2} U_{k}}+\epsilon\right)\right] I_{k} \\
& +\left[\lambda \frac{k}{\langle k\rangle}\left\langle k\left(U_{k}+\epsilon\right)\right\rangle+\gamma_{1}\left(\frac{\gamma_{2} U_{k}}{1+\gamma_{2} U_{k}}+\epsilon\right)\right]\left[\frac{(\alpha-\beta) U_{k}+\delta}{\alpha+\delta}+\epsilon\right] .
\end{aligned}
$$

Since $\epsilon>0$ is arbitrary small, we get

$$
\limsup _{t \rightarrow \infty} I_{k}(t) \leq \frac{\left[\lambda \frac{k}{\langle k\rangle}\left\langle k U_{k}\right\rangle+\frac{\gamma_{1} \gamma_{2}\left\langle U_{k}\right\rangle}{1+\gamma_{2}\left\langle U_{k}\right\rangle}\right]\left[\frac{\delta+(\alpha-\beta) U_{k}}{\alpha+\delta}\right]}{\beta+\lambda \frac{k}{\langle k\rangle}\left\langle k U_{k}\right\rangle+\frac{\gamma_{1} \gamma_{2}\left\langle U_{k}\right\rangle}{1+\gamma_{2}\left\langle U_{k}\right\rangle}} .
$$

Similarly, we can prove inequality (28).

Denote $\triangle_{k}=\left\{\left(I_{k}, R_{k}\right) \mid 0 \leq I_{k}+R_{k} \leq 1, I_{k} \geq 0, R_{k} \geq 0\right\}, k=1,2, \ldots, n$, and $\triangle=\prod_{k=1}^{n} \triangle_{k} \times$ $[0,1]$.

Theorem 3 If $\lambda>\lambda_{c}$ and $\alpha \geq \beta$, then system (2) has a unique endemic equilibrium $E^{*}=$ $\left\{I_{1}^{*}, R_{1}^{*}, \ldots, I_{n}^{*}, R_{n}^{*}, V^{*}\right\}$ which is of global attraction in $\Delta-\left\{F^{*}\right\}$, where $F^{*}=\left\{0, \frac{\alpha}{\alpha+\delta}, 0, \frac{\alpha}{\alpha+\delta}\right.$, $\left.\ldots, 0, \frac{\alpha}{\alpha+\delta}, 0\right\}$ is the disease-free equilibrium of system (2).

Proof Define a map $\mathcal{G}=\left\{\mathcal{G}_{1}, \mathcal{G}_{2}, \ldots, \mathcal{G}_{n}\right\}: \mathbb{R}^{n} \mapsto \mathbb{R}^{n}$ as follows:

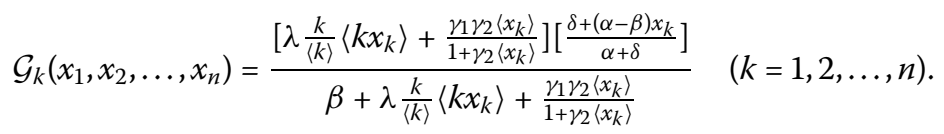

Let $U_{k}^{(1)}=1$ for all $k=1,2, \ldots, n$ and $U_{k}^{(m+1)}=\mathcal{G}_{k}\left(U_{1}^{(m)}, U_{2}^{(m)}, \ldots, U_{n}^{(m)}\right)$, it is obvious that $\lim \sup _{t \rightarrow \infty} I_{k}(t) \leq U_{k}^{(1)}=1$. According to Proposition 2, we then obtain

$$
\limsup _{t \rightarrow \infty} I_{k}(t) \leq U_{k}^{(m)}, \quad k=1,2, \ldots, n, m=1,2, \ldots
$$

Moreover, for all $k=1,2, \ldots, n$, we can testify the convergence of the sequences $\left\{U_{k}^{(m)}\right\}_{m=1}^{+\infty}$ by induction. First, it is obvious that $U_{k}^{(2)}<U_{k}^{(1)}=1$. Secondly, if $U_{k}^{(m+1)} \leq U_{k}^{(m)}$, then the reader can easily verify that $U_{k}^{(m+2)} \leq U_{k}^{(m+1)}$. It implies that the sequence $\left\{U_{k}^{(m)}\right\}$ is convergent. Denoted by $U_{k}=\lim _{m \rightarrow \infty} U_{k}^{(m)}$, we then have $\limsup _{t \rightarrow \infty} I_{k}(t) \leq U_{k}, k=1,2, \ldots, n$.

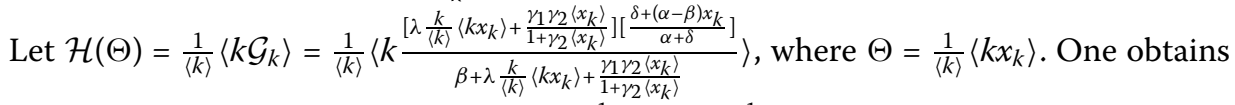
that $\left.\mathcal{H}^{\prime}(\Theta)\right|_{\Theta=0}>1$ if $\lambda>\lambda_{c}$. It implies that $\frac{1}{\langle k\rangle}\left\langle k \mathcal{G}_{k}\right\rangle>\frac{1}{\langle k\rangle}\left\langle k x_{k}\right\rangle$ when $x_{k}>0(k=1,2, \ldots, n)$ is small enough.

Denote $L_{k}^{(m+1)}=\mathcal{G}_{k}\left(L_{1}^{(m)}, L_{2}^{(m)}, \ldots, L_{n}^{(m)}\right)$ for each $k=1,2, \ldots, n$. According to Lemma 1 in paper [14], we can take $L_{k}^{(1)}$ small enough such that $\forall k, 0<L_{k}^{(1)}<\liminf _{t \rightarrow \infty} I_{k}(t)$ and $L_{k}^{(2)}>$ $L_{k}^{(1)}$. If $L_{k}^{(m)} \geq L_{k}^{(m-1)}$, it is easy to testify that $L_{k}^{(m+1)} \geq L_{k}^{(m)}$. In result, the sequences $\left\{L_{k}^{(m)}\right\}_{m=1}^{+\infty}$ are convergent for all $k$, and denote $L_{k}=\lim _{m \rightarrow \infty} L_{k}^{(m)}$.

Both $\left\{L_{k}\right\}$ and $\left\{U_{k}\right\}$ satisfy the following equation:

$$
I_{k}=\frac{\left[\lambda \frac{k}{\langle k\rangle}\left\langle k I_{k}\right\rangle+\gamma_{1} \frac{\gamma_{2}\left\langle I_{k}\right\rangle}{1+\gamma_{2}\left\langle I_{k}\right\rangle}\right]\left[\frac{\delta+(\alpha-\beta) I_{k}}{\alpha+\delta}\right]}{\beta+\lambda \frac{k}{\langle k\rangle}\left\langle k I_{k}\right\rangle+\gamma_{1} \frac{\gamma_{2}\left\langle I_{k}\right\rangle}{1+\gamma_{2}\left\langle I_{k}\right\rangle}} .
$$


After some transformations, we can find that both $\left\{L_{k}\right\}$ and $\left\{U_{k}\right\}$ satisfy Eq. (7). Thus one obtains that $U_{k}=L_{k}=I_{k}^{*}$ and $\lim _{t \rightarrow \infty} I_{k}(t)=I_{k}^{*}$ according to the uniqueness of endemic equilibrium of system (1). The proof of Theorem 3 is completed.

\section{Simulations}

In the section above, some theoretical results for the proposed mean-field equations are revealed. For a finite size network, there exists an epidemic threshold $\lambda_{c}$, if the infection rate $\lambda<\lambda_{c}$, then the disease-free equilibrium is globally asymptotically stable. Otherwise, the disease-free equilibrium is unstable and a unique globally attracting endemic equilibrium exists.

In this section, we will perform some numerical simulations to confirm the theoretical results over BA (Barabási-Albert) scale-free networks which are generated by the preferential attachment algorithm [27]. All the networks used in the simulations were built using $N=10^{4}$ nodes.

The epidemics are seeded with randomly chosen fraction of nodes to avoid stochastic extinction. The probability of a susceptible node with $n(\leq$ degree $k$ ) infectious neighbors being infected in small interval of time $h$ is $1-(1-\lambda h)^{n}\left(1-\gamma_{1} h V(t)\right)$ at step $t+1$. The term $(1-\lambda h)^{n}$ represents the probability that a susceptible node cannot be infected by his (her) infectious neighbors, and the term $1-\gamma_{1} h V(t)$ represents the probability that a susceptible node cannot be infected by infectious vectors. In this paper, we give the numerical simulations from different angles:

- The epidemic threshold $\lambda_{c}$ that changes as functions of different model parameters.

- The final density of the infected nodes that changes as functions of different model parameters.

In stochastic simulations, the dynamics are totally evolved for 2,000 time steps, we set the time interval $h=0.1$ and let $\rho=\frac{1}{T} \sum_{t=t_{0}}^{t_{0}-1+T} \rho(t)$ (here, $T=100, t_{0}=1,901$ ) be the time average to reduce the fluctuation of $\rho(t)$. At the same time, to minimize random fluctuation caused by the initial conditions, we make average of $\rho$ over 100 realizations of different initial infectious nodes. Let $\lambda$ increase systematically by $\Delta \lambda$ beginning with $\lambda=0$, if $\rho>0.0005$ as $\lambda=\lambda^{*}$ and $\rho<0.0005$ as $\lambda<\lambda^{*}$, we set $\lambda_{c}=\lambda^{*}-\Delta \lambda$.

In Figure 1, we illustrate the variation of the epidemic threshold $\lambda_{c}$ with respect to the parameters $\gamma_{1}$ and $\gamma_{2}$ both for stochastic simulations (SS) and also for mean-field (MF)

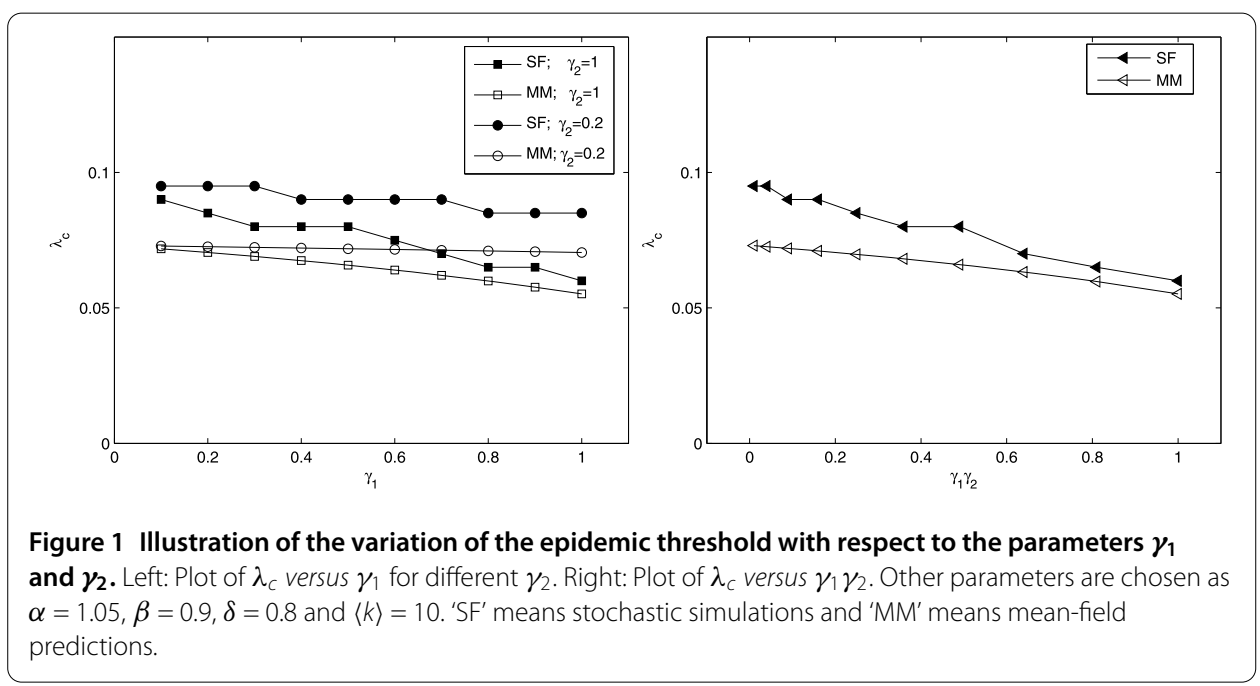



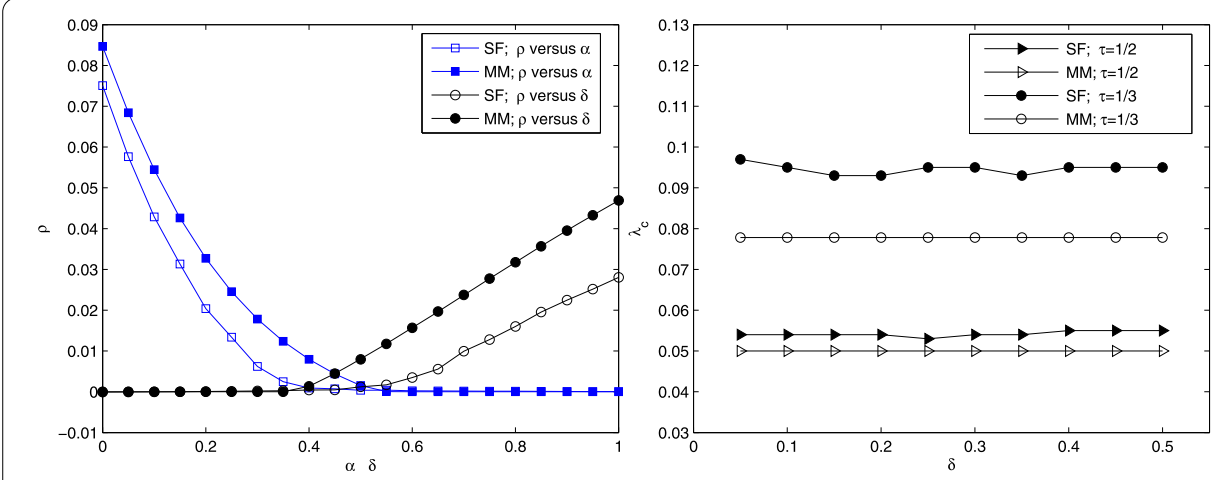

Figure 2 Illustration of the variations of the final infected density and the epidemic threshold with respect to the parameters $\alpha$ and $\delta$. Left: Plot of $\rho$ versus $\alpha$ and $\delta$. When considering $\rho$ versus $\alpha$, we set $\delta=0.5$; when considering $\rho$ versus $\delta$, we set $\alpha=0.4$. Right: The variation of the infection threshold $\lambda_{c}$ with respect to $\delta$ over BA scale-free network for different $\tau=\frac{\delta}{\alpha+\delta}$. Other parameters are chosen as $\gamma_{1}=\gamma_{2}=0.6$, $\beta=0.8$ and $\langle k\rangle=10$.

predictions formula (18). It is clear that the epidemic threshold $\lambda_{c}$ decreases as $\gamma_{1}\left(\right.$ or $\left.\gamma_{2}\right)$ increases. We thus conclude that reducing the contacts between individuals and vectors can effectively control the spread of the disease over networks. From this angle, the stochastic simulations agree well with the mean-field predictions. The discrepancy between these is also shown in our simulations, we can see the stochastic simulations are slightly larger than the theoretical predictions for threshold $\lambda_{c}$, which is likely to be due to a distribution cutoff effect on a finite size network [28] and other neglected factors (for instance, network is static and has degree-correlations). Moreover, one can find that for larger infection rates $\gamma_{1}$ and $\gamma_{2}$, the error between stochastic simulation and theoretical predictions is smaller, which is likely to be attributed to the contacts homogeneity between individuals and vectors.

Figure 2 illustrates the variations of the final infected density $\rho$ (left) and the epidemic threshold $\lambda_{c}$ (right) with respect to parameters $\alpha$ and $\delta$, respectively. It is clear that the discrepancy remains for the effect of a finite size network. The theoretical prediction of formula (18) shows that for different parameters $\alpha$ and $\delta$, the epidemic threshold $\lambda_{c}$ is unchanged as long as $\tau=\frac{\delta}{\alpha+\delta}$ is unchanged. From Figure 2 we can see that the stochastic simulations are in accordance with mean-field predictions disregarding the slight fluctuations for the effect of the stochastic factor. Also, one can see that the larger direct immunization rate $\alpha$, the larger the epidemic threshold is from Figure 2 (right). Namely, the direct immunization can increase the critical threshold of epidemic spreading on complex networks and reduce the prevalence of infectious disease, which agrees with the results of paper [24]. Consequently, we can prevent the disease spreading by improving immunization strength.

Figure 3 reflects the relation between the final densities of infected nodes and infection rate $\lambda$ for different average degree. One can see that the larger average degree, the lower the discrepancy rate is. We simulate the time series of total densities of infected nodes on the BA network with $\langle k\rangle=20$ in Figure 4 . We can obtain that the epidemic threshold $\lambda_{c}=0.032$ from formula (18). It is clear that if $\lambda<\lambda_{c}$, the disease will disappear quickly; otherwise $\lambda>\lambda_{c}$ the disease will persist in this system. Considering the factor that the epidemic threshold $\lambda_{c}$ of stochastic simulations is larger than the one of the mean-field 

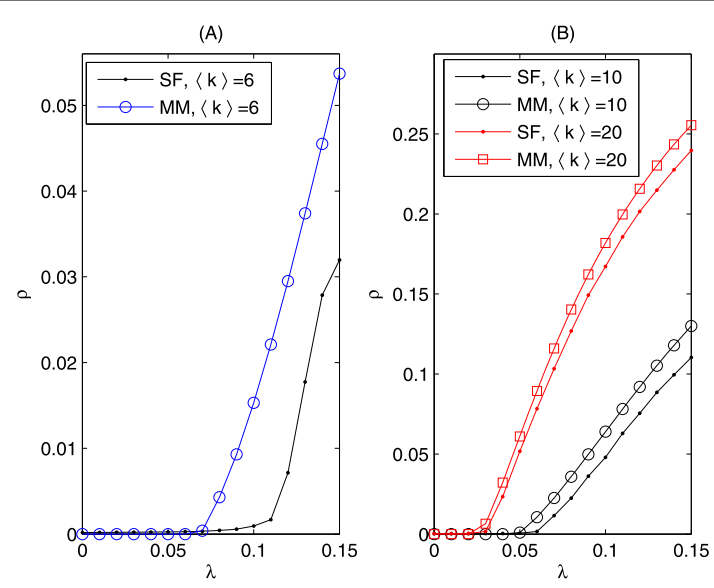

Figure 3 The variation of the stable densities of infected nodes with respect to infection rate $\lambda$ for BA scale-free network, where $\alpha=0.83, \beta=0.7, \delta=0.71, \gamma_{1}=0.45, \gamma_{2}=0.54$.

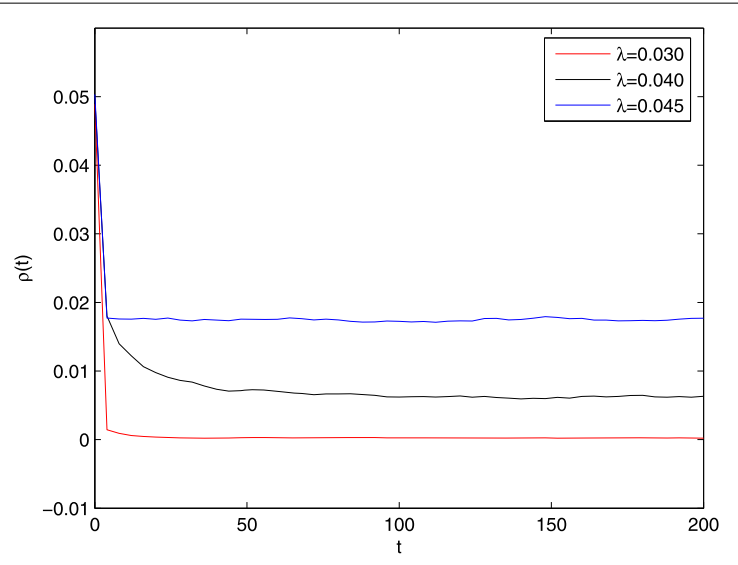

Figure 4 The total densities of infected nodes that change as time series on BA scale-free network with $\langle k\rangle=20$, where $\alpha=0.83, \beta=0.8, \delta=0.7, \gamma_{1}=0.60, \gamma_{2}=0.60$.

predictions, we think the stochastic simulations are reasonably consistent with the theoretic results.

\section{Conclusions}

In order to better explain the mechanism of spreading of epidemics, we have investigated a novel SIRS model with direct immunization via two infection mechanisms in this paper. The model is approximately described by the mean-field method neglecting contact duration.

The disease-free equilibrium and endemic equilibrium and their dynamics are discussed in this paper. Our theoretic results show that for finite size networks, there exists epidemic threshold $\lambda_{c}$, below which the disease-free equilibrium is globally asymptotically stable; otherwise a unique endemic equilibrium exists. We prove theoretically that the endemic equilibrium is of attraction when $\lambda>\lambda_{c}$ under the assumption that $\alpha \geq \beta$. To go a step further, we perform some numerical simulations to test and verify our theoretical results. 
From simulations as above, we can find that the discrepancies between stochastic simulations and theoretical predictions remain for the effect of a finite size network [28] and stochastic factors. Disregarding these slight errors, we think that the numerical simulations confirm to the theoretical results, and the mean-field approach is of effectiveness. Especially, one can see that the larger transmission rates from infected vectors to susceptible individuals $\gamma_{1}$ or from infected individuals to susceptible vectors $\gamma_{2}$, the better the simulations accord with the mean-field predictions. Just as mentioned above, this may attribute to the homogeneity of contacts between individuals and vectors.

\section{Competing interests}

The authors declare that they have no competing interests.

\section{Authors' contributions}

All authors contributed equally to the writing of this paper. All authors read and approved the final manuscript.

\section{Author details}

${ }^{1}$ College of Sciences, Jiujiang University, Qianjin East Road, Jiujiang, 332005, China. ${ }^{2}$ School of Mathematics and Computing Science, Guilin University of Electronic Technology, Guilin, 541004, China.

\section{Acknowledgements}

This research was jointly supported by NSFC Grant 11401274 and the Natural Science Foundation of Jiangxi Province (Grants 20142BDH80027 and 20132BAB201012). RZ Yu was also supported by the Foundations of Jiangxi Education Bureau (Grants GJJ12617 and GJJ13714).

Received: 10 October 2014 Accepted: 3 March 2015 Published online: 09 April 2015

\section{References}

1. Pastor-Satorras, R, Vespignani, A: Epidemic spreading in scale-free networks. Phys. Rev. Lett. 86, 3200-3203 (2001)

2. Pastor-Satorras, R, Vespignani, A: Epidemic dynamics and endemic states in complex networks. Phys. Rev. E 63, 066117 (2001)

3. Yang, M, Chen, GR, Fu, XC: A modified SIS model with an infective medium on complex networks and its global stability. Physica A 390, 2408-2413 (2011)

4. Pastor-Satorras, R, Vespignani, A: Epidemic dynamics in finite size scale-free networks. Phys. Rev. E 65, 035108 (2002)

5. d'Onofrio, A: A note on the global behavior of the network-based SIS epidemic model. Nonlinear Anal., Real World Appl. 9, 1567-1572 (2008)

6. Fu, X, Small, M, Walker, DM, Zhang, H: Epidemic dynamics on scale-free networks with piecewise linear infectivity and immunizations. Phys. Rev. E 77, 036113 (2008)

7. Xia, C, Wang, Z, Sanz, J, Meloni, S, Morenoc, Y: Effects of delayed recovery and nonuniform transmission on the spreading of diseases in complex networks. Physica A 392, 1577-1585 (2013)

8. Wang, L, Dai, GZ: Global stability of virus spreading in complex heterogeneous networks. SIAM J. Appl. Math. 68, 1495-1502 (2008)

9. Yang, R, Wang, BH, Ren, J, Bai, WJ, Shi, ZW, Xu, W, Zhou, T: Epidemic spreading on heterogeneous networks with identical infectivity. Phys. Lett. A 364, 189-193 (2007)

10. Li, K, Small, M, Zhang, H, Fu, X: Epidemic outbreaks on networks with effective contacts. Nonlinear Anal., Real World Appl. 11, 1017-1025 (2010)

11. Ball, F, Neal, P: Network epidemic models with two types of mixing. Math. Biosci. 212, 69-87 (2008)

12. Volz, E: SIR dynamics in random networks with heterogeneous connectivity. J. Math. Biol. 56, $293-310$ (2008)

13. Kiss, IZ, Green, DM, Kao, RR: The effect of contact heterogeneity and multiple routes of transmission on final epidemic size. Math. Biosci. 203, 124-136 (2006)

14. Lou, J, Ruggeri, T: The dynamics of spreading and immune strategies of sexually transmitted diseases on scale-free network. J. Math. Anal. Appl. 365, 210-219 (2010)

15. Miller, JC: A note on a paper by Erik Volz: SIR dynamics in random networks. J. Math. Biol. 62, 349-358 (2011)

16. Xia, C, Wang, L, Sun, S, Wang, J: An SIR model with infection delay and propagation vector in complex networks. Nonlinear Dyn. 69, 927-934 (2012)

17. Valdez, LD, Macri, PA, Braunstein, LA: Intermittent social distancing strategy for epidemic control. Phys. Rev. E 85 036108 (2012)

18. Wu, Q, Fu, X, Small, M, Xu, X-J: The impact of awareness on epidemic spreading in networks. Chaos 22, 013101 (2012)

19. Molesworth, AM, Cuevas, LE, Connor, SJ, Morse, AP, Thomson, MC: Environmental risk and meningitis epidemics in Africa. Emerg. Infect. Dis. 9(10), 1287-1293 (2003)

20. Wang, Y, Jin, Z, Yang, Z, Zhang, Z-K, Zhou, T, Sun, G-Q: Global analysis of an SIS model with an infective vector on complex networks. Nonlinear Anal., Real World Appl. 13, 543-557 (2012)

21. Shi, HJ, Duan, ZS, Chen, GR: An SIS model with infective medium on complex networks. Physica A 387, 2133-2144 (2008)

22. Cooke, KL: Stability analysis for a vector disease model. Rocky Mt. J. Math. 9, 31-42 (1979) 
23. Busenberg, S, Cooke, KL: Periodic solutions of a periodic nonlinear delay differential equation. SIAM J. Appl. Math. 35, 704-721 (1978)

24. Xia, C, Liu, Z, Chen, Z, Yuan, Z: SIRS epidemic model with direct immunization on complex networks. Control Decis. 23, 468-472 (2008) (in Chinese)

25. Diekmann, O, Heesterbeek, JAP, Roberts, MG: The construction of next-generation matrices for compartmental epidemic models. J. R. Soc. Interface 7(47), 873-885 (2010)

26. Diekmann, O, Heesterbeek, JAP, Roberts, MG: On the definition and the computation of the basic reproduction ratio $R_{0}$ in models for infectious diseases in heterogeneous populations. J. Math. Biol. 28, 365-382 (1990)

27. Barabási, AL, Albert, R: Emergence of scaling in random networks. Science 286, 509-512 (1999)

28. Olinky, R, Stone, L: Unexpected epidemic thresholds in heterogeneous networks: the role of disease transmission. Phys. Rev. E 70, 030902(R) (2004)

Submit your manuscript to a SpringerOpen ${ }^{\odot}$ journal and benefit from:

- Convenient online submission

Rigorous peer review

- Immediate publication on acceptance

- Open access: articles freely available online

- High visibility within the field

- Retaining the copyright to your article 